\title{
A confusing case of multiple sclerosis and central nervous system graft versus host disease
}

\author{
Byung-Nam Yoon ${ }^{1}$, Choong Kun $\mathrm{Ha}^{1}$, Kwang-Woo Lee ${ }^{2}$, Sung-Hye Park ${ }^{3}$, and Jung-Joon Sung ${ }^{2}$
}

${ }^{1}$ Department of Neurology, Inha University Hospital, Incheon; Departments of ${ }^{2}$ Neurology and ${ }^{3}$ Pathology, Seoul National University Hospital, Seoul, Korea

Received: March 12, 2015

Revised : April 28, 2015

Accepted: May 3, 2015

\section{Correspondence to}

Jung-Joon Sung, M.D.

Department of Neurology, Seoul

National University Hospital,

101 Daehak-ro, Jongno-gu, Seoul

03080 , Korea

Tel: $+82-2-2072-2820$

Fax: +82-2-3672-7553

E-mail:jjsaint@snu.ac.kr

\section{To the Editor,}

The neurologic manifestations observed in allogeneic stem cell transplantation (allo-SCT) recipients are related to infections, metabolic abnormalities, and relapse of malignant disease in the central nervous system (CNS). After undergoing allo-SCT, graft-versus-host disease (GVHD) can become a serious problem that contributes to patient morbidity. GVHD is a systemic syndrome mediated by donor $\mathrm{T}$ cells and principally targets the skin, gut, and liver. Involvement of other organ systems has been described. Several reports have described that GVHD affects the CNS [1,2]; however, CNS-GVHD is a rare finding and remains controversial. Confounding factors, including infections, drugs, and autoimmune manifestations can make the diagnosis challenging. Among the reports, only a few small case reports have described histological findings. We experienced a case in which the symptoms mimicked those of CNS-GVHD after allo-SCT but were ultimately considered a newly developed case of multiple sclerosis (MS).

A 36-year-old man with no history of demyelinating disease or any other specific illness was diagnosed with acute lymphoblastic leukemia. The patient underwent matched related allo-SCT. Cyclosporin-A followed by tacrolimus were administered for GVHD prophylaxis. Approximately 8 months after al-
lo-SCT, he was admitted to our hospital complaining of watery diarrhea. An endoscopic biopsy of the colon revealed multiple apoptotic bodies in the crypts and increased lymphoplasma cell infiltration in lamina propria, and he was diagnosed with gastrointestinal GVHD (GI-GVHD). High-dose oral steroids were administered and improved his symptoms. No eventful or GVHD-related disease was observed for 1 year after allo-SCT. Additionally, his bone marrow showed normal blood cells and complete donor chimerism. The patient presented 2 years after allo-SCT with a progressive tingling sensation below the abdomen and weakness in both legs. He also described progressive difficulty climbing stairs and walking. He did not complain of visual disturbances, dizziness, or headache. A neurological examination demonstrated symmetrical muscle weakness in both legs (Medical Research Council [MRC] grade 3) and increased deep tendon reflexes during induced knee and ankle reflexes. A sensory examination revealed decreases in all modalities below the T6 dermatome. Normal results were observed on the mini-mental state examination, as well as on cranial nerve and cerebellar function tests. No Babinski sign or ankle clonus were observed. Brain and spinal magnetic resonance imaging (MRI) revealed high T2 signal intensity lesions at $T_{3}$ level of the spinal cord and of the 


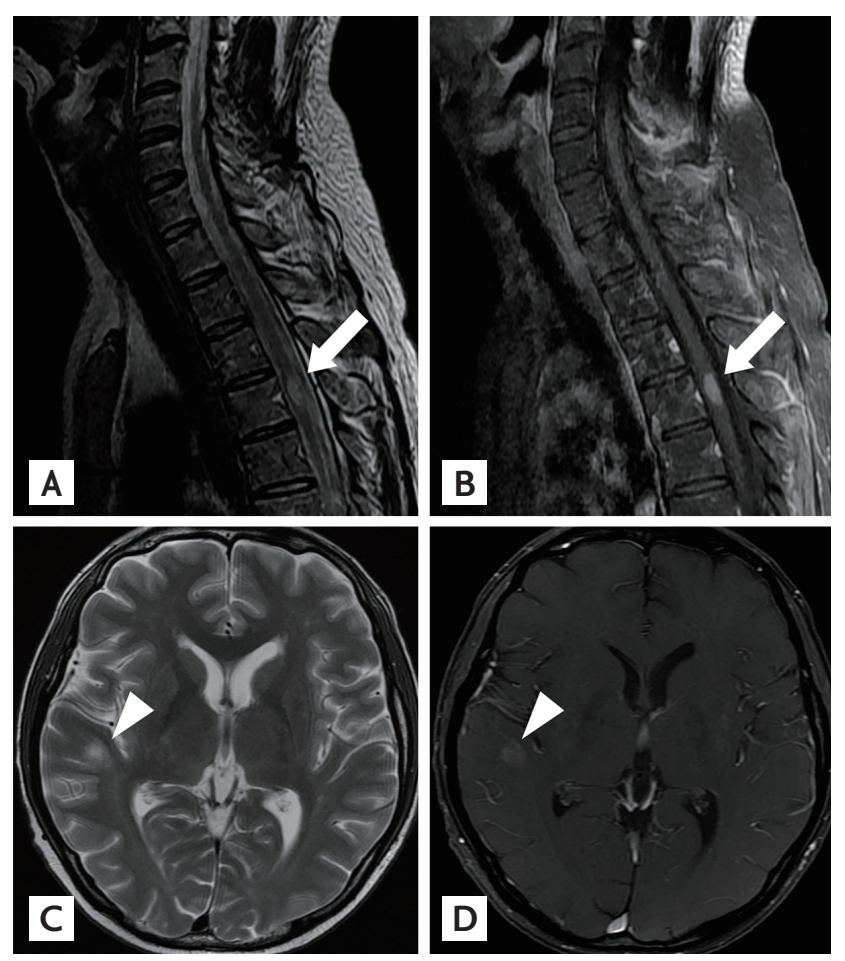

Figure 1. Magnetic resonance imaging (MRI) scans of the spine and brain. (A, B) Sagittal T2-weighted MRI of the thoracic cord demonstrates an intramedullary enhancing lesion at $\mathrm{T}_{3}$ (arrows). (C, D) Axial T2-weighted MRI of the brain shows round hyperintense lesions with slight enhancement in the right temporal subcortex (arrowheads).

corpus callosum and right temporal subcortex (Fig. 1). These lesions were enhanced with gadolinium. Cerebrospinal fluid (CSF) was clear and showed no pleocytosis, oligoclonal bands, or increased protein. Serum and CSF viral studies were negative for Epstein-Barr virus (EBV), cytomegalovirus, and the John Cunningham virus. There was no evidence of a CNS infection or leukemia relapse in the CNS. Furthermore, he had a GI-GVHD history; thus, we diagnosed probable CNS-GVHD. The patient received high dose intravenous methylprednisolone for 5 days followed by oral prednisolone (6o mg). Despite this treatment, his symptoms failed to improve. A stereotactic brain biopsy (right temporal lobe) revealed gitter cells (foamy macrophages) and loss of myelinated nerve fibers without leukemic infiltration (Fig. 2). An excision biopsy of the thoracic spinal cord revealed only fibrosis. His symptoms had improved gradually 3 weeks later. Strength in the lower extremities was MRC 4 and sensory numbness showed steady improvement. The patient was discharged and could ambulate independently. Oral

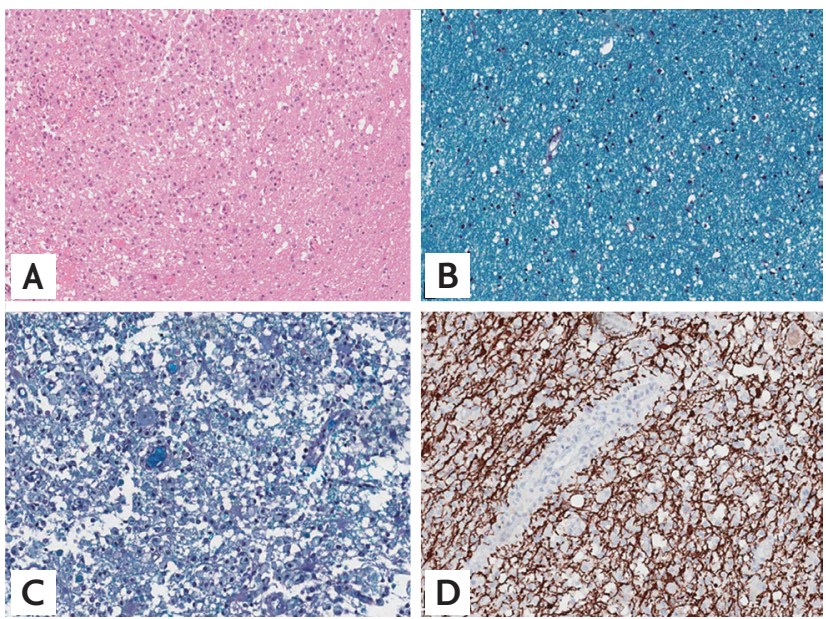

Figure 2. Histology of the brain biopsy. (A) The lesion shows a large number of gitter cells (foamy macrophages) infiltrating with a relatively sharp border with the normal area $(\mathrm{H} \& \mathrm{E}, \times 5 \mathrm{O})$. (B) Normal area shows intact myelinated nerve fibers on Luxol fast blue (LFB) staining ( $\times 200)$. (C) The lesion shows near-complete loss of myelinated nerve fibers with heavy macrophage infiltration compared to the normal area (LFB stain, $\times 200)$. (D) Neurofilament stain $(\times 100)$ of the lesion shows relatively intact axons.

prednisolone was tapered by $15 \mathrm{mg}$ every other day over 8 months. The patient redeveloped a tingling sensation and weakness in all extremities approximately 3 years after allo-SCT. Neurological signs indicated weakness in bilateral arms (MRC grade 3 ) and legs (MRC grade 2) and decreased sensory responses below the Ti spinal cord level, and increased bilateral deep tendon reflexes in the upper and lower extremities. A spinal MRI revealed partially enhanced $\mathrm{C} 1-2$ lesions in the spinal cord (Fig. 3). Anti-nuclear antibody, anti-neutrophil cytoplasmic antibody, rheumatoid factor, anti-Ro, anti-La, cryoglobulin antibody, and anti-aquaporin-4 antibody were not detected. Both P1oo latencies were delayed (right, $120 \mathrm{~ms}$; left, $125 \mathrm{~ms}$ ) in a visual evoked study. Furthermore, electrophysiologic evidence suggested central conduction delays in bilateral median and posterior tibial nerve evoked potentials. He was treated with high doses of intravenous methylprednisolone, and his symptoms improved gradually. Arm and leg weakness had become MRC 51 month later. We initially considered CNS-GVHD as a possible diagnosis based on the CNS characteristics. We could have also considered a CNS demyelinating disease, which can cause relapsing myelopathy, such as relapsing and remitting MS, as another possible diagnosis. The patient was started on subcutaneous injections of inter- 


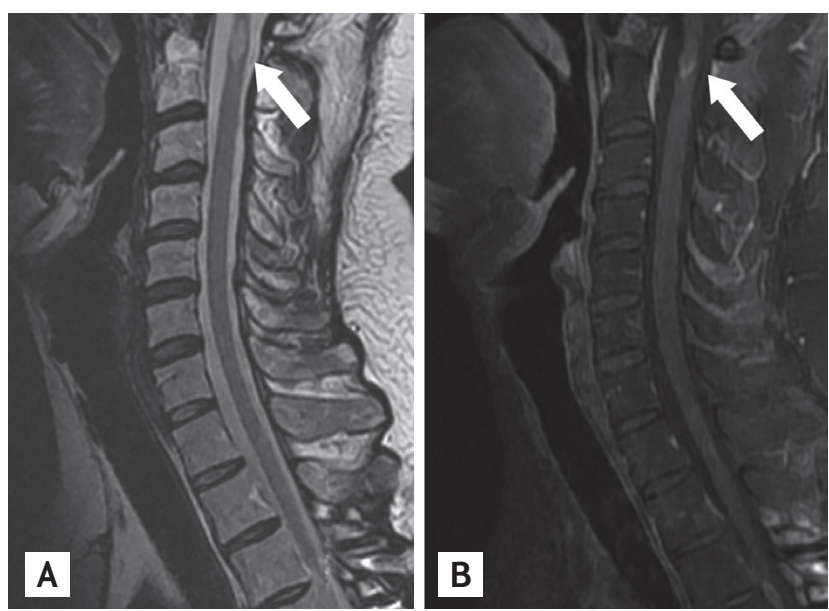

Figure 3. Magnetic resonance imaging (MRI) scans of the cervical spine (secondary bilateral lower leg weakness 3 years after allogeneic stem cell transplantation). (A, B) Sagittal T2-weighted MRI of the cervical cord demonstrates an intramedullary enhancing lesions at $\mathrm{C}_{1}$ and $\mathrm{C}_{2}$ (arrows). The previous enhancing lesion (at $\mathrm{T}_{3}$ ) disappeared.

feron $\beta$ (IFN- $\beta$ ) in addition to oral prednisolone (10 $\mathrm{mg})$. No eventful symptoms were detected that indicated a relapse during a 36-month observation period.

Although GVHD commonly affects the skin, gastrointestinal tract, liver, and hematopoietic system, some reports have described patients with CNS manifestations associated with GVHD and support the possibility that the brain is a target organ for GVHD based on animal studies [3]. However, among the reported antemortem diagnoses of possible CNS-GVHD, few had histological corroboration. This entity is particularly difficult to prove because differential diagnoses, such as CNS lymphoma, opportunistic infection, and EBV-related disorders, must be excluded. CNS-GVHD should be considered only if there is no evidence of other diseases with overlapping features, radiographic characteristics of CNS involvement, response to immunosuppressive therapy, and adequate documentation of a perivascular T-cell infiltrate after a histological and immunophenotypic evaluation.

The evaluation for infectious disease was negative in our patient. The histological results were not compatible with relapsed leukemia in the CNS. The patient developed GI-GVHD, as demonstrated by an endoscopic biopsy, and developed relapsing myelopathy and asymptomatic brain lesions. The initial diagnosis was CNS-GVHD; however, we considered MS as a probable diagnosis for several reasons. First, the immunohistochemistry of the brain biopsy was negative for infiltration of cytotoxic T-cells (CD+3) or apoptosis. Near-complete loss of myelinated nerve fibers was observed using Luxol fast blue stain, and axons were relatively spared. The pathological analysis of the brain biopsy was more compatible with a CNS demyelinating disease than with CNS-GVHD. Second, GI-GVHD affects only the CNS and does not involve other organs for approximately 5 years after onset. Third, MRI imaging revealed multiple white matter brain and spinal cord lesions. These separate episodes of neurological symptoms and radiological findings are characteristic of MS. Last, relapses continued to occur despite sufficient steroid treatment, whereas no relapses were observed for 36 months after the patient received IFN- $\beta$. IFN- $\beta$ specifically targets MS and induces a more effective response against MS as compared to GVHD. Therefore, its application may be an important was to distinguish the two suspected diseases.

Autoimmune disorders may increase in incidence after allo-SCT. Myasthenia gravis, dermatomyositis, polymyositis, Guillain-Barre syndrome, and acute disseminated encephalomyelitis with an autoimmune component have been reported in patients after a bone marrow transplant (BMT). Demyelination disorders after BMT are very uncommon and only a few cases have been reported [4]. Histological findings suggestive of GVHD have been confirmed in a minority of reported cases, and the exact etiology of the demyelination remains largely undefined.

Immunotherapies, such as cyclophosphamide, mitoxantrone, chemotherapy, and allo-SCT have been evaluated to assess their efficacy for treating refractory MS, which affects only a small portion of patients with MS. Allo-SCT has been suggested as a potential treatment for MS and other autoimmune disorders that fail to respond to approved therapies. In contrast, our patient was a case of newly developed MS after allo-SCT. Because this was such a rare finding, we report it as an exemplary case. It is unfortunate that we have no information about whether the donor had a demyelinating disorder or developed MS after donating bone marrow.

One case study describing recurrent CNS manifestations after allo-SCT has been reported [5]. However, all cases were considered CNS-GVHD rather than MS based on clinical symptoms and histological findings. Distinguishing between CNS-GVHD and MS can be 
challenging, as the two diseases share similar clinical presentations and radiological findings. Furthermore, both CNS-GVHD and MS are rare in a clinical setting. However, the consequences of both diseases can be quite serious. Continued reporting and work-up of similar cases is required to develop strategies for a timely and accurate differential diagnosis.

Keywords: Stem cell transplantation; Multiple sclerosis; Graft vs host disease

\section{Conflict of interest}

No potential conflict of interest relevant to this article was reported.

\section{REFERENCES}

1. Marosi C, Budka H, Grimm G, et al. Fatal encephalitis in a patient with chronic graft-versus-host disease. Bone Marrow Transplant 1990;6:53-57.

2. Shortt J, Hutton E, Faragher M, Spencer A. Central nervous system graft-versus-host disease post allogeneic stem cell transplant. Br J Haematol 2006;132:245-247.

3. Padovan CS, Gerbitz A, Sostak P, et al. Cerebral involvement in graft-versus-host disease after murine bone marrow transplantation. Neurology 2001;56:1106-1108.

4. Denier C, Bourhis JH, Lacroix C, et al. Spectrum and prognosis of neurologic complications after hematopoietic transplantation. Neurology 2006;67:1990-1997.

5. Matsuo Y, Kamezaki K, Takeishi S, et al. Encephalomyelitis mimicking multiple sclerosis associated with chronic graft-versus-host disease after allogeneic bone marrow transplantation. Intern Med 2009;48:1453-1456. 